\title{
Compact Laser-Diode Sources for Optical Inspection Probes
}

\author{
MARK R. BACHHUBER AND ROBERT D. LORENZ, MEMBER, IEEE
}

\begin{abstract}
Noncontacting optical inspection probes offer the potential to improve the flexibility and accuracy of manufacturing systems. One goal in this area is the development of compact and lightweight optical probe heads to make them more practical for manufacturing systems such as robotics, where size and weight are important. One solution to meet this goal is to use laser diode sources rather than conventional tube lasers to provide the illumination beam used to make optical measurements. Laser diode sources can provide coherent, stable illumination for many optical probe applications if a number of technical issues are first addressed. These issues include temperature control, output intensity control, and collimation of the light output. These technical issues will be addressed and possible solutions, as well as present experimental results from a laboratory optical probe developed using a laser diode source, will be presented.
\end{abstract}

\section{INTRODUCTION}

$\mathrm{A}^{\mathrm{N}}$ NUMBER of probes that use the principles of optical triangulation have been developed since the mid 1970's. Successful application of optical triangulation for optical probes first required the development of suitable laser sources and position-sensing photodetectors. Appropriate lasers have been available since the early 1960's while appropriate photodetectors were not available until the mid 1970's. The early lasers developed were primarily gas tube lasers while the position sensing photodetectors developed included lateraleffect photodiodes and linear photodiode arrays.

Fig. 1 shows the basic model for an optical triangulation probe. The probe parameters can be defined as follows.

$l_{t} \quad$ is the distance along the illumination beam from the optical null to the illuminated point on the target surface.

$d_{00}$ is the distance along the optical axis from the imaging lens to the lens' secondary focal point.

$d_{0}$ is the distance from the illuminated point on the target surface to the imaging lens.

$d_{i}$ is the distance along the optical axis from the imaging lens to the image on the detector.

$P_{i}$ is the distance along the linear detector, from the

Paper GID 89-3, approved by the Machine Tools, Robotics, and Factory Automation Committee of the IEEE Industry Applications Society for presentation at the 1987 Industry Applications Society Annual Meeting, Atlanta, GA, October 19-23. Manuscript released for publication January 5 1989.

M. R. Bachhuber is at 931 Meadow Lane, Mayville, WI 53050.

R. D. Lorenz is with the Mechanical, Electrical, and Computer Engineering Department, University of Wisconsin-Madison, 1513 University Avenue, Madison, WI 53706-1691.

IEEE Log Number 8927821.

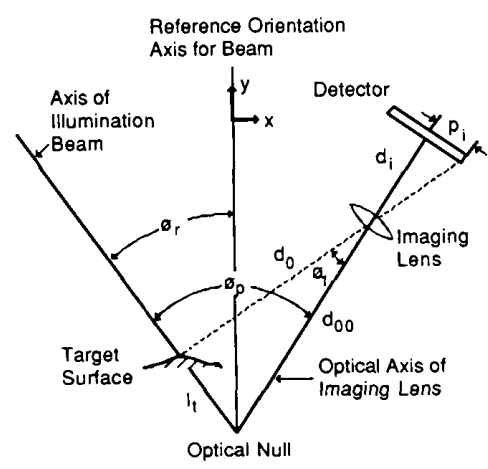

Fig. 1. Model of optical triangulation probe.

intersection of the optical axis with the detector surface to the center of the image formed on the detector.

$\phi_{1}$ is the angular deviation of $d_{0}$ relative to $d_{00}$.

$\phi_{p}$ is the probe's included angle between the illumination beam and the lens optical axis.

$\phi_{r}$ is the orientation angle of the illumination beam to an arbitrary reference axis in the triangulation plane [1], [2].

As the target surface moves, the image of the illumination spot formed on the detector moves across the detector, and thus changes in target surface position can be computed as a function of the known probe geometry.

A number of factors must be considered in the design of optical triangulation probes to achieve suitable performance as well as the desired physical dimensions and characteristics. The geometric parameters described in the preceding can be manipulated to provide an infinite number of probe configurations with different ranges to resolution ratios. Other factors that can affect probe resolution are the characteristics of the illumination beam and the ability to resolve image position for a given photodetector. A particular probe design can be optimized to give a relatively compact configuration for an optical probe head. Allowances for the size of the components used can be a major factor in the final probe head dimensions.

One of the major components of the optical probe is the laser source used. Conventional tube lasers can provide stable, coherent illumination but are inherently large relative to achievable probe head dimensions. Conventional tube lasers are also disadvantageous in applications where light intensity levels are crucial for measurement resolution. This is because 
conventional tube lasers must employ an optomechanical attenuation means to control light intensity. This increases the number of components needed and therefore the size and weight of the probe.

A practical solution to the problems associated with tube lasers is to use diode lasers as the probe source. Diode laser sources can also provide stable coherent illumination but are much more compact devices. In addition to their size and weight advantages, the diode lasers' light-intensity levels can be modulated by controlling the laser diode current. However, there are also a number of technical issues which must be resolved before laser diode sources can be used effectively. The output characteristics of laser diodes are very temperature-dependent, and unlike conventional tube lasers, which emit a coherent well-collimated beam of light, their output power is poorly collimated. Therefore a temperature controller must be used to regulate the laser-diode spectral content as well as an output intensity controller to regulate the laser diode current. Finally, suitable collimating optics must also be used to reduce beam divergence.

\section{Laser Diode Sources}

The first diode lasers capable of continuous wave $(\mathrm{CW})$ operation at room temperature were developed in the early 1970 's. Since this time, numerous improvements have been made, so that today commercially available diode lasers can be obtained with a variety of output characteristics. Maximum output powers can be obtained from a few milliwatts to as much as $40 \mathrm{~W}$. Emitted wavelengths can be obtained from approximately 700 to $1300 \mathrm{~nm}$. Furthermore, most diode lasers can operate in either a $\mathrm{CW}$ or pulsed mode. Diode lasers can lase in a number of optical modes, but many provide stable operation in the fundamental $T E_{00}$ mode.

The temperature dependence of diode laser properties is an important consideration in achieving satisfactory operation for many applications. For a given operating current, a temperature rise will cause the output power of the diode laser to decrease and the peak wavelength emitted to shift by a small amount. Typical temperature coefficients for these properties are $0.2 \mathrm{~mW} / \mathrm{C}^{\circ}$ and $0.2 \mathrm{~nm} / \mathrm{C}^{\circ}$, respectively.

With a suitable temperature controller developed, the output power of the diode laser can be modulated by regulating the current. Fig. 2 illustrates the output characteristics at a constant temperature and as a function of diode laser current. As the diagram shows, the current must first reach a certain threshold value before the device will start to lase. Below this threshold value, the laser diode behaves like an LED, emitting light with a relatively large spectral content.

The final consideration when implementing laser diodes is the form of the optical output. Fig. 3 shows the typical farfield pattern for a diode laser. As the figure illustrates, the divergence angle can change depending on the orientation with respect to the junction plane in a laser diode crystal. In applications where a well-collimated illumination beam is required, appropriate optical components must be used to reduce this beam divergence.

If each of the aforementioned operation characteristics is properly addressed, the diode laser can provide stable,

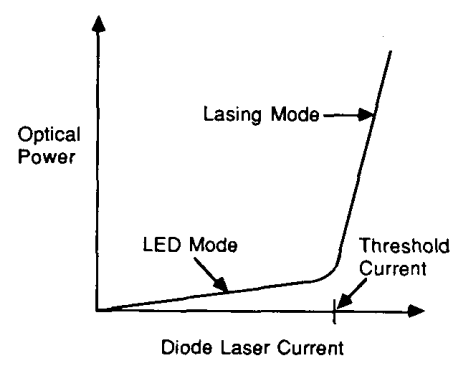

Fig. 2. Light output of diode laser as function of current.

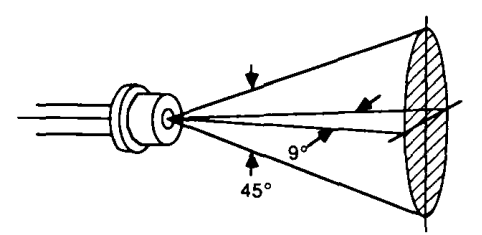

Fig. 3. Schematic representation of far-field emission of typical diode laser.

coherent, and well-collimated light output similar to a conventional tube laser for optical probe applications.

\section{Temperature Controller}

The cooling of the laser diode must be designed to maintain the goal of a flexible and compact source for use in optical probe heads. One form of cooling which can provide an effective yet compact method of temperature control is thermoelectric cooling. Thermoelectric coolers are solid-state devices which function as heat pumps, similar to conventional refrigeration systems. These heat pumps are also refered to as Peltier coolers since they rely primarily on the Peltier effect to derive their cooling ability. The Peltier effect is the evolution or absorption of heat at the junction of two dissimilar materials (over and beyond Joule heat) accompanying a flow of current [3]. Thermoelectric coolers are available in many sizes and with many different heat-pumping capacities. The physical dimensions of the coolers can be as small as 2 or $3 \mathrm{mms}$ to a side, and heat pumping capacities can range from only a few tenths of a watt to well over $50 \mathrm{~W}$ [4].

To formulate an appropriate control strategy for the temperature of the laser diode, knowledge of the thermal system dynamics must be known. The thermal system dynamics can be estimated by deriving a suitable model for the diode-laser thermal system. From this model, the system dynamics and controller performance can be evaluated.

The temperature controller used must provide adequate temperature stabilization to ensure that appropriate intensity levels are obtained from the diode laser or to ensure that the spectral content of the light emission remains stable. The controller must be able to respond to temperature disturbances arising from the diode laser itself or from the surrounding environment. The diode laser should be maintained at a suitable temperature level to ensure a normal operation lifetime and to prevent catastrophic failure.

The control strategy for a laser-diode thermal system that 
will be presented here is a state-variable control strategy that has five general steps:

- writing out the state variable form of the system differential equations,

- drawing the state variable form of the block diagram,

- identifying the physical system state feedback gain terms,

- setting up a parallel structure of controller state feedback gain terms having the same physical dimensions as the physical system state feedback gain terms,

- applying block diagram or matrix state equations analysis to establish the desired physical system equivalent closedloop state feedback gain terms [5]

The primary elements to be modeled for the thermal system are the diode laser and the thermoelectric cooler. In addition to these elements, two additional models will be used to represent temperature-sensing devices which will provide feedback for the case temperature of the diode laser and the cold-side temperature of the thermoelectric cooler. Each of these components will be represented using a lumped parameter model, which assumes a uniform temperature. Therefore each element can be considered as a thermal mass with a thermal capacitance. From a system diagram of each of the components, the thermodynamic relationships between the diode laser, thermoelectric cooler, and temperature sensors can be determined. For the system described here the thermoelectric cooler is physically attached to the diode laser and a temperature sensor is physically attached to both the cooler and the laser diode. Applying the state-variable approach, the state variable form of the energy balance equation for each thermal mass can be written as follows:

$$
\begin{gathered}
C_{E} \frac{d T_{E}}{d t}=-\left(B_{E}+R_{1}+R_{2}\right) T_{E}+R_{1} T_{L}+Q_{\text {in }} \\
C_{L} \frac{d T_{L}}{d t}=R_{1} T_{E}-\left(B_{L}+R_{1}+R_{2}\right) T_{L}+R_{2} T_{T 1} \\
C_{T 1} \frac{d T_{T 1}}{d t}=R_{2} T_{L}-\left(B_{T 1}+R_{2}\right) T_{T 1} \\
C_{T 2} \frac{d T_{T 2}}{d t}=R_{3} T_{E}-\left(B_{T 2}+R_{3}\right) T_{T 2}
\end{gathered}
$$

where

$C_{E}, C_{L}, C_{T 1}, C_{T 2}$ are the thermal capacitances of the thermoelectric cooler, diode laser, laser temperature sensor, and cooler temperature sensor, respectively;

$T_{E}, T_{L}, T_{T 1}, T_{T 2}$ are the component temperatures;

$R_{1}, R_{2}, R_{3} \quad$ account for contact resistances between the laser and cooler, the laser and its temperature sensor, and the cooler and its temperature sensor, respectively;

$B_{E}, B_{L}, B_{T 1}, B_{T 2} \quad$ account for thermal losses of the components with the environment;

$Q_{\text {in }} \quad$ is the power input to the thermoelectric cooler.

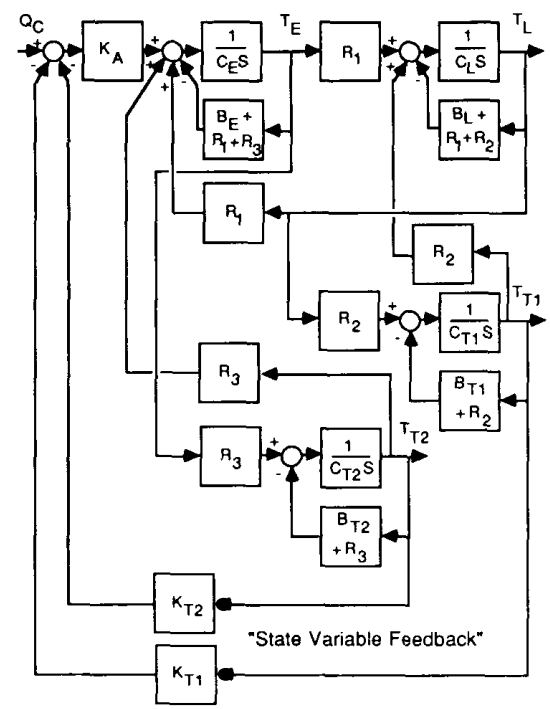

Fig. 4. State-variable form of block diagram.

Fig. 4 illustrates the state-variable form of the block diagram for the thermal system described in the preceding, with the state-variable feedback terms already included. The physical-state feedback gain terms are the cooler temperature $T_{E}$ and the diode laser temperature $T_{L}$. Since the statefeedback gain terms $K_{T 1}$ and $K_{T 2}$ cannot be applied directly to these quantities in the physical system, they are applied to the temperatures $T_{T 1}$ and $T_{T 2}$ measured by the temperature sensors.

With the state-variable model developed, an analysis can be made to determine how the state-feedback gain terms should be tuned to achieve the best system performance. A simulation of the aforementioned model showed that by increasing the inner-state feedback loop gain $K_{T 2}$ applied to the cooler temperature sensor, the step response of the thermoelectric cooler temperature could be increased significantly, although the diode laser response remained relatively unchanged. With $K_{T 2}$ tuned to achieve optimum performance for the cooler temperature response, the outer state-feedback loop gain, $K_{T 1}$, can be tuned to achieve optimum performance for the diodelaser temperature response.

The state-variable approach makes tuning much easier for systems such as the diode-laser temperature controller since each loop being tuned has only one gain to adjust, unlike PI or PID controllers, where the gains are interdependent. The ease of tuning makes this approach simpler to implement in a laboratory or field setting. Furthermore, in many cases the controller-state feedback gains are significantly larger than the physical-state feedback gains, making the estimates of the physical gains less critical, thereby improving controller robustness.

\section{OUtPut INTENSITY CONTROLLER}

With a stable operating temperature, the output intensity of a diode laser will be primarily a function of the applied current. The performance characteristics of the intensity 


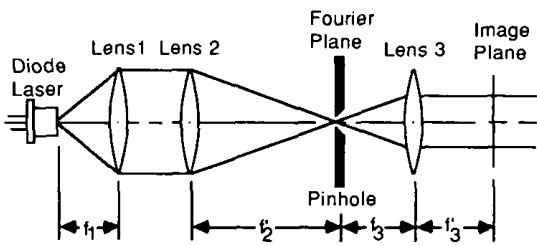

Fig. 5. Diode laser optical system for collimation.

controller developed will be dependent on whether $\mathrm{CW}$ or pulsed operation is desired. Intensity feedback information can often be obtained from a pin photodiode. which is commonly incorporated into the diode laser package. Another source of intensity feedback can be the position-sensing photodetector used in the optical probe. The intensity controller dynamics can be set to obtain the desired output characteristics using proportional control.

For $\mathrm{CW}$ operation. a stable current above the lasing threshold must be applied. Intensity feedback information from a $p-i-n$ photodiode in the laser package can be used to maintain a stable intensity level as well to insure that maximum output intensity levels are not exceeded. Outside the loop closed by a $\mathrm{p}-\mathrm{i}-\mathrm{n}$ photodiode, the intensity level seen at the probe photodector can be used to adjust the operating point for optimal probe performance.

For pulsed operation, bandwidths in excess of $1 \mathrm{GHz}$ can be achieved with most commercially available diode lasers, although greater care must be taken in the design of the drive electronics so that the circuit elements do not limit the circuit's modulation bandwidth. For very high modulation rates, the operating current must be biased above the lasing threshold to achieve the best performance.

\section{Collimation Optics}

Since most optical probes will require the collimation of the diode-laser output power, appropriate collimating optics must be used. For an optical triangulation probe such as the one presented in this paper, the characteristics of the illumination beam are crucial in determining the accuracy of the optical probe measurements. The intensity distribution of the illumination beam should have a Gaussian-like shape similar to the illumination beam from a conventional tube laser.

Fig. 5 illustrates a possible optical system for collimation of the output power. Lens 1 and lens 2 serve as light collection and refocusing lenses, respectively. Using the principles of Fourier optics, the location of the pinhole can be shown to be a Fourier plane of the system, which means that an optical image will be transformed into its spatial frequency spectrum. If the light emitted from the laser diode is considered a point source, the light intensity pattern formed at the pinhole will be what is commonly known as the Airy pattern. The pinhole can then be used as a spatial filter to block the high spatial frequency components in the intensity pattern as well as any scattered light due to lens imperfections. The light emerging from the pinhole can then be collimated by lens 3 to produce an illumination beam with the desired size and a smooth Gaussian-like intensity distribution.

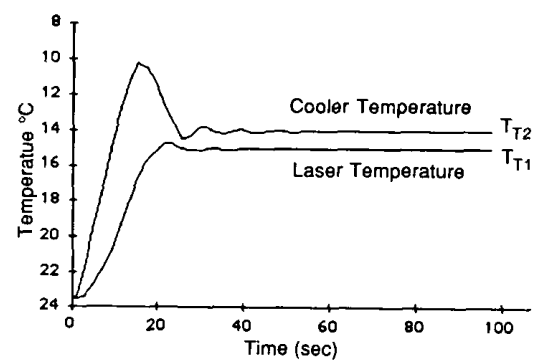

Fig. 6. Closed-loop step response curves for thermal system.

The optical components for the aforementioned optical system can be chosen with suitable focal lengths to maintain the compactness of the diode laser source for an optical probe head. In applications where an illumination beam with a smooth intensity distribution is not critical, the filtering optics may not be necessary, thereby making the optical system even more compact.

\section{Experimental Optical Triangulation Probe using a Laser Diode Source}

The technical issues and possible solutions to them presented up to this point were used to apply the diode laser source to an optical triangulation probe in the laboratory. A suitable diode laser was chosen and a temperature and an output intensity controller were developed and implemented to show that the diode laser could be successfully substituted for conventional HeNe tube lasers. Technical specifications for the devices selected are provided in an Appendix.

\section{Temperature Controller Implementation}

The thermoelectric module for the cooling system was chosen so it would operate at or near its optimum cooling level. The module selected had a maximum heat-pumping capacity of $4 \mathrm{~W}$ operating at a current of $3.5 \mathrm{~A}$. The temperature sensors chosen were thermistors, which were attached to the diode laser and thermoelectric cooler that formed the thermal system. A state-variable controller was then implemented and tuned to optimize its performance. Fig. 6 shows the actual step response curves obtained after both state feedback loops were tuned. The temperature of the diode laser using this controller was observed to remain stable to about $0.1^{\circ} \mathrm{C}$ at an operating point of approximately $21^{\circ} \mathrm{C}$.

\section{Output Intensity Controller Implementation}

The diode laser was chosen to have an output power similar to the 2-mW HeNe tube lasers commonly used in the laboratory. A Mitsubishi ML-3401 diode laser was chosen with a maximum output power of $3 \mathrm{~mW}$ and a lasing threshold current of approximately $20 \mathrm{~mA} \mathrm{[6].} \mathrm{This} \mathrm{diode} \mathrm{laser} \mathrm{operates}$ in the fundamental mode and is capable of either $\mathrm{CW}$ or pulsed operation. This particular diode laser also had a pin photodiode incorporated into the laser package for monitoring the output intensity.

A proportional controller was used to stabilize the optical output, using feedback from the pin photodiode. The diode 


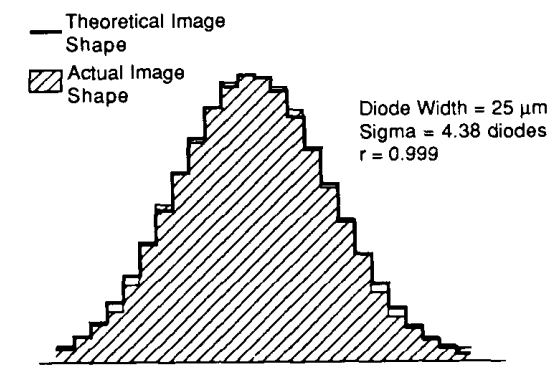

Fig. 7. Illumination beam and fitted Gaussian shape for diode laser.

laser was operated in a $\mathrm{CW}$ mode with the operating point adjusted manually, depending on the optical probe measurement conditions. The output intensity controller was found to be stable to approximately $0.02 \mathrm{~mW}$ and thus provided the stable optical output that would be needed to make accurate measurements. To prevent the possibility of any transients which could cause the diode laser output to exceed maximum ratings, a slower starter circuit was also implemented between the power supply and diode laser circuit.

\section{Collimation of Laser-Diode Output Power}

The controlled diode laser source was next incorporated into an optical bench setup using optical slides and positioners to construct a variety of optical triangulation probe configurations. First, the output of the diode laser was collimated using the optical system described previously. A $200-\mu \mathrm{m}$ pinhole was used to filter the optical image after the collection/ focusing optics. The size of the pinhole was selected to allow only the low-frequency components of the intensity pattern to pass. A third lens (lens 3 ) was then used to produce the final illumination beam.

Fig. 7 shows the shape of the illumination beam as seen by a 1024 linear photodiode array. As the figure shows, the illumination beam obtained had a Gaussian-like shape similar to the shape of the illumination beam for tube lasers. The beam was observed to have a full angle divergence on the order of 1 mrad, and the shape appeared to remain constant along the beam.

\section{Optical Bench Tests of Experimental Optical Probe}

The optical bench setup was next configured to simulate an optical triangulation probe. An optical probe configuration was chosen to demonstrate that a compact configuration was possible while still maintaining a relatively high range-toresolution ratio. With a probe angle $\phi_{p}$ of $23^{\circ}$, a number of tests were run to determine the measurement accuracy and repeatability of the laser-diode optical probe. The target surface used as the reference measurement was a micrometer $x-y$ positioning stage with a positioning resolution of 0.001 inch and a range of 0.7 inch. Measurement test runs were made over short ranges at 0.001 -inch intervals and over the entire range of the positioning stage at 0.025 -inch intervals. The repeatability for each of these runs was also tested to demonstrate that the laser-diode optical probe could make stable measurements. Figs. 8 and 9 show the linearity and

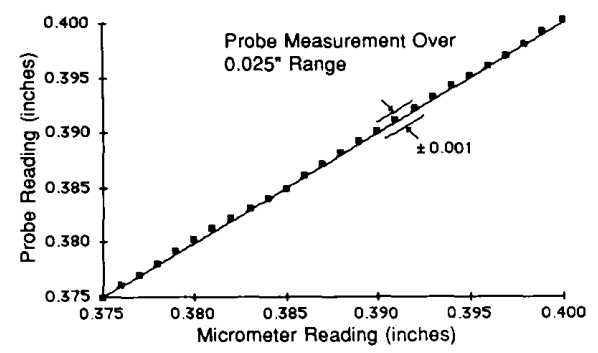

Fig. 8. Short-range linearity test.

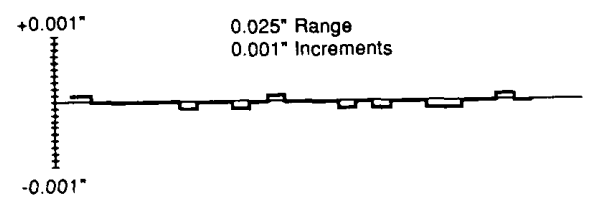

Fig. 9. Short-range repeatability test.

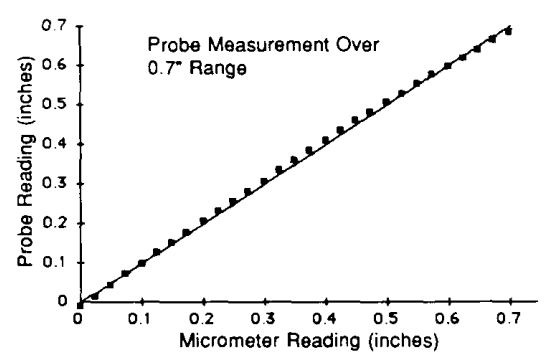

Fig. 10. Long-range measurement test.

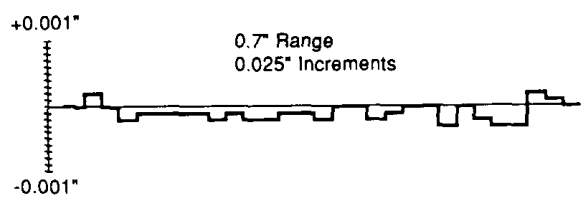

Fig. 11. Long-range repeatability test.

repeatability of the probe over a 0.025 -inch range. Figs. 10 and 11 show the linearity and repeatability over a 0.7 -inch range.

The test results show that the laser-diode optical triangulation probe could make accurate measurements down to the resolution of the micrometer positioning stage. Although optical triangulation theory predicts resolution down to 0.0001 inch for this configuration, the limit of the probe resolution could not be conclusively determined due to the limited resolution of the micrometer stage. The ability to modulate the output intensity of the laser diode made it possible to easily adjust the output intensity for different target surfaces to maintain the signal level at an optimum on the probe detector.

\section{ConCLusion}

The application of diode laser sources for optical inspection probes has been presented and demonstrated on an optical 
triangulation probe. The following conclusions can be drawn relative to the diode laser, its use, and the optical probe source.

- The state-variable temperature controller developed will allow operation of diode lasers when intensity level changes are needed or when spectral content of the illumination beam is important.

- The output intensity controller will allow stable operation of the diode laser as well as provide an electronic means for controlling the light-intensity level required. This eliminates the need for optomechanical variable attenuators, which are needed for conventional tube lasers.

- Laboratory experiments have shown that the diode laser can be substituted for a tube laser while maintaining the desired performance, thereby allowing the size of optical probe heads to be reduced considerably. Position experiments have shown that resolution greater than 0.001 inch can be obtained with a repeatability of \pm 0.0002 inch.

- Since diode lasers are available with a wide range output powers, there is more flexibility in choosing a laser to suit the measurement conditions.

\section{APPENDIX}

Techilcal. Specifications of the Selected Devices

\section{Thermoelectric (T.E.) Cooler Specifications}

Device:

Number of T.E. elements:

Length/area of T.E. element:

Seebeck coefficient:

Resistivity of elements:

Thermal conduction coefficient:

Melcor module CP1.4-17-10L.

$$
N_{e}=34 .
$$$$
\lambda=13.015 \mathrm{~cm} \cdot 1 \text {. }
$$$$
S=2.10510^{-4} \mathrm{~V} /{ }^{\circ} \mathrm{K}
$$$$
\rho=1.07610^{-3} \Omega \cdot \mathrm{cm} \text {. }
$$$$
k=1.66210^{-2} \mathrm{~W} /\left(\mathrm{cm}^{\circ} \mathrm{K}\right) .
$$

\section{Calculated Operating Point Characteristics}

Cold-side temperature of module: $\quad T_{c}=293^{\circ} \mathrm{K}$.

Heat sink temperature:

Optimum heat pumped:

Optimum current:

Optimum voltage:

$$
\begin{aligned}
& T_{h}=298^{\circ} \mathrm{K} . \\
& Q_{\mathrm{opt}}=0.258 \mathrm{~W} . \\
& I_{\mathrm{opt}}=0.237 \mathrm{~A} . \\
& V_{\mathrm{opt}}=0.149 \mathrm{~V} .
\end{aligned}
$$

\section{Thermistor Specifications}

Device:

Operating resistance at $294^{\circ} \mathrm{K}: \quad 8.44 \mathrm{k} \Omega$

Resistance coefficient at $294^{\circ} \mathrm{K}: 0.51 \mathrm{k} \Omega /{ }^{\circ} \mathrm{K}$.

\section{Laser Diode Specifications}

Device: Mitsubishi ML-3401.

$$
\underline{\text { Maximum Ratings }}
$$

Light output (CW):

Reverse voltage (laser diode):

Reverse voltage (photodiode):

Forward current:

Operating case temperature range: $15 \mathrm{~V}$. $10 \mathrm{~mA}$. -40 to $+60^{\circ} \mathrm{C}$.

\section{Typical Operating Characteristics at $25^{\circ} \mathrm{C}$}

Threshold current CW:

$20 \mathrm{~mA}$.

Lasing wavelength:

$850 \mathrm{~nm}$.

Full angle at half maximum power points:

$\theta_{\perp}$

$\theta_{\|}$

Operating current (CW, 3-mW output):

$13^{\circ}$.

$38^{\circ}$.

$30 \mathrm{~mA}$.

$1.8 \mathrm{~V}$.

$\begin{array}{ll}\text { Reverse current photodiode }\left(V_{R D}=10 \mathrm{~V}\right): & 0.5 \mu \mathrm{A} . \\ \text { Capacitance (photodiode): } & 7 \mathrm{pF} .\end{array}$

Monitoring output current

(CW, 3-mW output, $V_{P D}=1 \mathrm{~V}, R_{P D}=10 \Omega$ ): $\quad 0.3 \mathrm{~mA}$.

\section{REFERENCES}

[1] R. D. Lorenz, "The theory and design of optical/electronic probes for high performance measurement of parts," Ph.D. dissertation, Univ. of Wisconsin-Madison, 1984.

[2] R. D. Lorenz, "A novel, high range-to-resolution ratio, optical sensing technique for high speed surface geometry measurements," in Proc. SPIE Cambridge Symp. on Advances in Intelligent Robotic Systems, Oct. 26-31, 1986.

[3] K. R. Rao, "Thermoelectric energy conversion," in Proc. Conf. on Thermoelectric Devices and Their Applications, Vol. 1, Electrical Engineering Dept., Univ, of Texas-Arlington, May 19-23, 1975.

[4] Application Notes for Thermoelectric Devices, Trenton, NJ: Melcor Product Literature, 1985.

[5] R. D. Lorenz, "Synthesis of state variable controllers for industrial servo drives," in Proc. Conf. on Applied Motion Control, Minneapolis, MN, June 1986

[6] Mitsubishi Laser Diode Product Literature, Sunnyvale, CA: Mitsubishi Electric Corp., 1986.

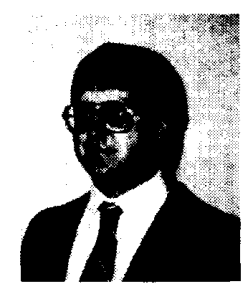

Mark R. Bachhuber received the B.S. and M.S. degrees in mechanical engineering, with controls/ electromechanics as his specialization area, from the University of Wisconsin-Madison, in 1984 and 1987, respectively.

Since 1987 he has been with Bachhuber Manufacturing Corporation in Mayville, WI, where he is presently the Engineering Manager. His responsibilities include automation and computer control aspects of their machinery and manufacturing processes.

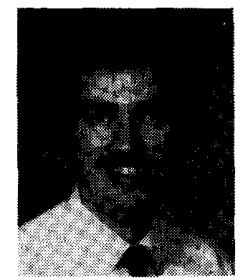

Robert D. Lorenz (S'83-M'84) received the B.S. M.S., and Ph.D. degrees, all in mechanical engineering, from the University of Wisconsin-Madison in 1969,1970 , and 1984 , respectively.

$\mathrm{He}$ is presently a member of the faculty of the University of Wisconsin-Madison, where he is an Assistant Professor of mechanical engineering and Affiliate Assistant Professor of electrical and computer engineering. In this position he acts as Director of the Advanced Automation and Robotics Consortium and as an Associate Director of the Wisconsin Electric Machines and Power Electronics Consortium. In the summer of 1987 he was a Visiting Professor at the Electrical Drives Institute of the Technical University of Aachen, West Germany. In 1969-1970 he did his master's thesis research at the Machine Tool Institute of that same university. From 1972 to 1982 he was a member of the research staff at the Gleason Works, in Rochester, NY. His current research interests include optical sensor and inspection technologies, real-time digital signal processing techniques, and ac drive and high-precision machine control technologies. He is a very active Consultant.

Dr. Lorenz is a member of the Machine Tools, Robotics, and Factory Automation Committee, the Industrial Drives Committee, and the Industria Control Committee of the IEEE Industry Applications Society. He is a Registered Professional Engineer in the States of New York and Wisconsin, 


\title{
An Adaptive Seam Tracker for Welding Heavy-Section Aluminum
}

\author{
ANDREW N. VAVRECK, NITIN NAYAK, EDWARD D. BUSHWAY III, AND ASOK RAY, SENIOR MEMBER, IEEE
}

\begin{abstract}
The process of welding aluminum presents a considerable challenge to the application of robotic welding. Heat distortion and fixturing errors often require alteration in welding parameters and the welding torch position and orientation in real-time. A nonadaptive robotic welding system, or a system with only limited sensory ability, is consequently unable to perform well in many aluminum welding tasks. A strong need exists in the aluminum fabrication industry for sophisticated flexible automated welding systems, which implement expert systems for weld process control and multipass weld planning, and apply advanced sensory technology for real-time seam-tracking and joint geometry measurement.
\end{abstract}

\section{INTRODUCTION}

$\mathrm{T}$ HE INTRODUCTION of robotic welding systems is underway in industry as part of a general move toward automating production lines. Robotic implementation is being initiated to satisfy a perceived need for high quality welds in shorter cycle times, and to acknowledge the recognized value of shifting the workforce from welding to sections of the production line with higher potential productivity and better environmental quality.

In general, robotic welding systems which cannot adapt in real-time to changes in the joint geometry along the weld seam and to the seam position itself have only limited success in many welding applications. The difficulty stems from heat distortion of the weldment or to an overall shift in the weld seam caused by fixturing errors, conditions often found in the welding of aluminum.

A solution to this problem requires the application of some form of adaptive joint geometry sensing system for guidance of the welding torch. The object of the research project described in this paper is to address the general aluminum welding problem by integrating a laser-based part-profiling sensor with a welding robot.

Paper GID 89-1, approved by the Machine Tools, Robotics, and Factory Automation Committee of the IEEE Industry Applications Society for presentation at the 1987 Industry Applications Society Annual Meeting, Atlanta, GA, October 19-23. This work supported by the Ben Franklin Partnership Program of the Commonwealth of Pennsylvania and BMY Division of HARSCO, Inc., York, PA. Manuscript released for publication January 5, 1989.

A. N. Vavreck is with the Manufacturing Science Office, Applied Research Lab, The Pennsylvania State University, P. O. Box 30, State College, PA 16804

N. Nayak and A. Ray are with the Mechanical Engineering Department The Pennsylvania State University, University Park, PA 16802.

E. D. Bushway III was with the Mechanical Engineering Department, The Pennsylvania State University, University Park, PA 16802; he is currently with the Space Products Division, Moog Incorporated, Jamison Road, Eas Aurora, NY 14052

IEEE Log Number 8927805

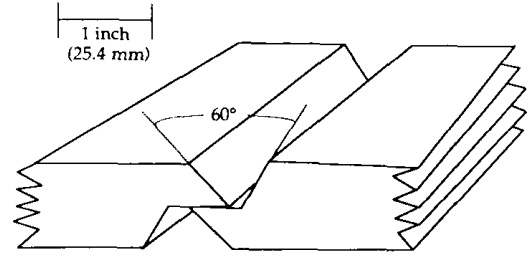

Fig. 1. Aluminum joint configuration

\section{Rationale for Adaptive Sensing}

The joint fit-up in aluminum, which is machined and wire brushed prior to welding, is good, but the seam placement and geometry can change significantly due to the input of heat from the welding process. In addition, aluminum is less forgiving to minor changes in the weld parameters than steel, and is much more susceptible to contamination. A typical aluminum heavy-section slip-joint weld seam is shown in Fig. 1.

An adaptive robotic welding system with seam-tracking sensors is necessary to weld typical aluminum sections, as a "blind" robotic welder is unable to correct for variations in seam placement and joint geometry and is unable to respond to movement of the joint due to heat distortion. The most commonly used adaptive welding strategy, and one which represents an inexpensive and robust technology, uses the arc current itself to guide the torch. The technique is known as through-arc sensing [1].

After manually teaching the robot the rough seam path, a low-current electrical signal through the welding wire is used to guide the robot to the root at the start of the seam. The arc is then turned on and the torch is moved along the taught path. The torch is not moved in a straight line along the path but instead is weaved from side to side. As the torch approaches the joint sides, the arc current increases. To guide the torch laterally through the seam, the center of the weave is continuously adjusted to make the current peaks level.

Typical through-arc systems can deal with complex seam geometries, and can perform multipass welds, with only a series of offsets required from the root pass to weld the remaining passes. Some systems can change the wirefeed rate or the torch speed to compensate for a varying root gap. A notable advantage of through-arc sensing is the fact that the position of the seam, via the torch, is measured directly, not at some distance ahead. This greatly reduces data processing time and enhances potential accuracy. The welding speed is not enhanced as a significant amount of speed is sacrificed by 